\title{
A Comparison of One-Way and Two-Way Coupling Methods for Numerical Analysis of Fluid-Structure Interactions
}

\author{
Friedrich-Karl Benra, Hans Josef Dohmen, Ji Pei, \\ Sebastian Schuster, and Bo Wan
}

Department of Mechanical Engineering, Turbomachinery, Faculty of Engineering Sciences, University of Duisburg-Essen, Lotharstraße 1, 47048 Duisburg, Germany

Correspondence should be addressed to Sebastian Schuster, sebastian.schuster@stud.uni-due.de

Received 13 January 2011; Revised 28 July 2011; Accepted 2 August 2011

Academic Editor: Edward Swim

Copyright (c) 2011 Friedrich-Karl Benra et al. This is an open access article distributed under the Creative Commons Attribution License, which permits unrestricted use, distribution, and reproduction in any medium, provided the original work is properly cited.

The interaction between fluid and structure occurs in a wide range of engineering problems. The solution for such problems is based on the relations of continuum mechanics and is mostly solved with numerical methods. It is a computational challenge to solve such problems because of the complex geometries, intricate physics of fluids, and complicated fluid-structure interactions. The way in which the interaction between fluid and solid is described gives the largest opportunity for reducing the computational effort. One possibility for reducing the computational effort of fluidstructure simulations is the use of one-way coupled simulations. In this paper, different problems are investigated with one-way and two-way coupled methods. After an explanation of the solution strategy for both models, a closer look at the differences between these methods will be provided, and it will be shown under what conditions a one-way coupling solution gives plausible results.

\section{Introduction}

The interaction between fluids and solids is a phenomenon that can often be observed in nature, for example, the deformation of trees or the movement of sand dunes caused by wind.

In almost the same manner, wind can interact with buildings, sometimes with dramatic consequences, such as the collapse of the Tacoma-Narrows Bridge in November 1940.

These processes can only be calculated using laws and equations from different physical disciplines. Examples like this, where the arising subproblems cannot be solved independently, are called multiphysics applications. A very important class of these multiphysics problems are fluid-structure interactions (FSIs), which are characterized by the fact that the flow around a body has a strong impact on the structure, and vice versa; the modification of the structure has a nonnegligible influence on the flow. Two disciplines 
involved in these kinds of multiphysics problems are fluid dynamics and structural dynamics, which can both be described by the relations of continuum mechanics.

On this note, FSI is a subset of multiphysics applications and is defined well by Zienkiewicz and Taylor [1]:

Coupled systems and formulations are those applicable to multiple domains and dependent variables which usually describe different physical phenomena and in which neither domain can be solved while separated from the other and neither set of dependent variables can be explicitly eliminated at the differential equation level.

Solution strategies for FSI simulations are mainly divided into monolithic and partitioned methods; this paper will focus only on partitioned methods. Information on partitioned methods can be found in the available literature [2-9], as well as information on mixed methods [10]. Partitioned methods are divided into one-way and two-way coupling. Two-way coupling is further divided into weakly and strongly coupled methods. Those interested in the differences between strongly and weakly coupled methods can find information in Vaassen et al. [11]. For instance, Pozarlik and Kok [12] compared one-way and two-way coupling results for the vibration of a combustion chamber.

\section{Numerical Approach}

Our investigations were performed using a partitioned method. On the fluid side, ANSYS CFX was used, and on the structure side, ANSYS Mechanical. ANSYS MFX coupling algorithm was used for the two-way coupled simulations.

\section{Calculations for the Fluid Side}

The solution of the fluid side is based on the continuity equation and the Navier-Stokes equation. The Navier-Stokes equation, written in Einstein summation convention, is given by (3.1). Further information about the solution for moving meshes can be found in Münsch and Breuer [13], especially the Arbitrary-Euler-Lagrange (AEL) formulation

$$
\rho \frac{\partial u_{i}}{\partial t}+\rho\left(\frac{\partial u_{i} u_{j}}{\partial x_{j}}\right)=-\frac{\partial p}{\partial x_{i}}+\eta \frac{\partial}{\partial x_{j}}\left(\frac{\partial u_{i}}{\partial x_{j}}+\frac{\partial u_{j}}{\partial x_{i}}-\frac{2}{3} \frac{\partial u_{k}}{\partial x_{k}} \delta_{i j}\right)
$$

A solution for this equation requires a fine discretization in space and time. In engineering problems, small scales are not within the scope of interest. To reduce the computational effort, the solution variables are split into a mean value and a fluctuation value, as shown in (3.2)

$$
\varphi=\bar{\varphi}+\varphi^{\prime}
$$

Using Reynolds averaging, we obtain (3.3), the Unsteady-Reynolds-Averaged-Navier-Stokes equation

$$
\rho \frac{\partial \bar{u}_{i}}{\partial t}+\rho\left(\frac{\partial \bar{u}_{i} \bar{u}_{j}}{\partial x_{j}}\right)=-\frac{\partial \bar{p}}{\partial x_{i}}+\eta \frac{\partial}{\partial x_{j}}\left(\frac{\partial \bar{u}_{i}}{\partial x_{j}}+\frac{\partial \bar{u}_{j}}{\partial x_{i}}-\frac{2}{3} \frac{\partial \bar{u}_{k}}{\partial x_{k}} \delta_{i j}\right)-\rho\left(\frac{\partial}{\partial x_{j}} \overline{u_{i}^{\prime} u_{j}^{\prime}}\right)
$$


In this equation, $\overline{u_{i}^{\prime} u_{j}^{\prime}}$ is the Reynolds stress tensor. Its value is calculated with turbulence models, such as the $k-\varepsilon, k-\omega$, or SST model.

The continuity equation and the Navier-Stokes equations are solved using a finite volume approach. Starting with the continuity equation in integral form (3.4), three terms must be solved: the first term describes the change in mass in the control volume; the second deals with the mass flux through the control volume boundary; the third describes the change of the control volume throughout mesh deformation

$$
\frac{d}{d t} \int_{V} \rho \cdot d V+\int \rho \cdot U_{j} \cdot d n_{j}+\int_{S} \rho \cdot W_{j} \cdot d n_{j}=0
$$

\section{Calculations for the Structure Side}

The calculations for the structure side are based on the impulse conservation (4.1). It is solved using a finite element approach, where a finite element is chosen for each specific problem

$$
M \cdot \overrightarrow{\ddot{u}} \cdot+C \cdot \overrightarrow{\dot{u}}+K \cdot \vec{u}=\vec{F}
$$

\section{Coupling}

Regardless of whether one-way or two-way coupling methods are used, the solutions are based on a partitioned method where separate solutions for the different physical fields are prepared. One field that has to be solved is fluid dynamics, the other is structure dynamics. At the boundary between fluids and solids, the fluid-structure interface, information for the solution is shared between the fluid solver and structure solver. The information exchanged is dependent on the coupling method. For one-way coupling calculations, only the fluid pressure acting at the structure is transferred to the structure solver. For two-way-coupling calculations, the displacement of the structure is also transferred to the fluid solver.

In Figure 1, on the top the solution procedure is shown for one-way coupling. Initially, the fluid field is solved until the convergence criteria are reached. The calculated forces at the structure boundaries are then transferred to the structure side. Next, the structure side is calculated until the convergence criterion is reached. Then, the fluid flow for the next time step is calculated to convergence. The solution is finished when the maximum number of time steps is reached.

The process flow chart for the strong two-way coupling algorithm is shown on the bottom of Figure 1. Within one-time step during the transient simulation, a converged solution for the flow field is required to provide the forces acting on the body. After interpolating the forces from the fluid mesh to the surface mesh of the structure, a converged solution of the structural dynamics will be attained under the effect of the acting forces. The response of the structure to the emerging load represents a displacement of the structural grid nodes. The displacements at the boundary between structure and fluid are interpolated to the fluid mesh which leads to its deformation. This step closes one inner loop of the simulation. For strong two-way-coupling simulations, these steps are repeated until the changes in the flow forces and the structural displacements fall below a prescribed amount. Afterwards, a new time step is launched. For weak two-way coupling simulations, the convergence at the boundary between structure and fluid is not considered and a new time step is launched directly. 


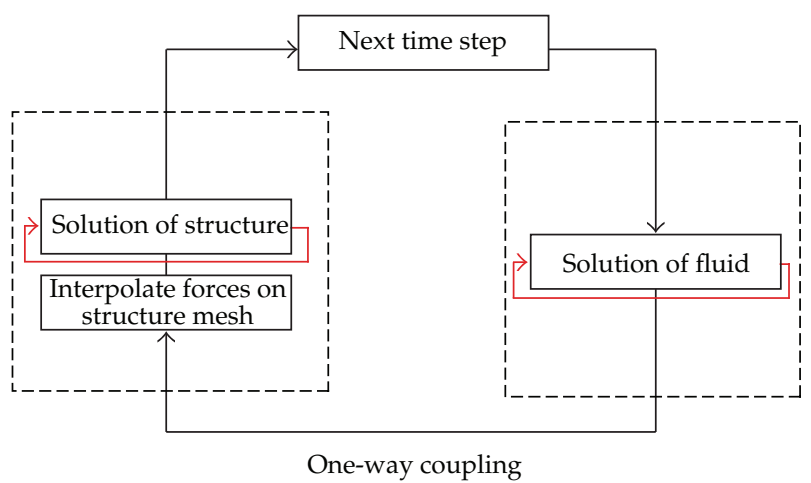

(a)

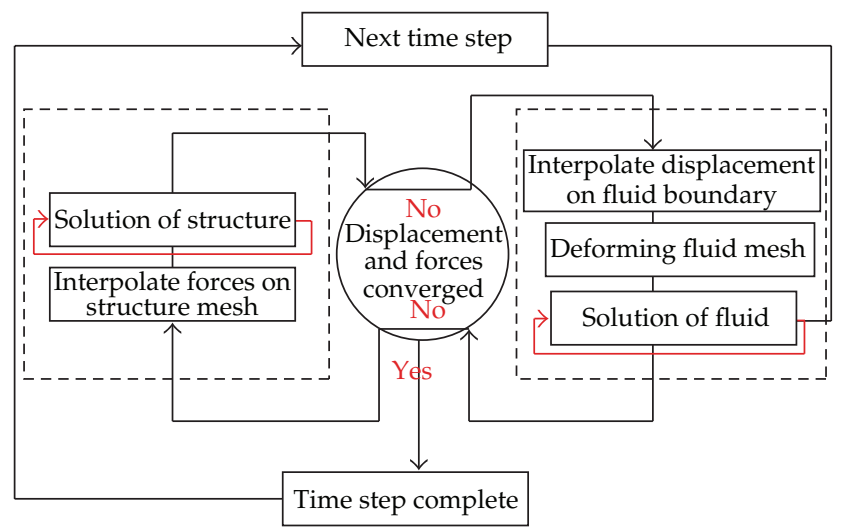

Strong two-way coupling

(b)

Figure 1: Solution algorithm for one- and strong two-way coupling.

Some general comments can be made about both methods. Often the advantage of one method is a disadvantage of the other. In general, the two-way coupling solution is more accurate, especially for larger deflections where the fluid field is strongly influenced by structural deformation. Strong two-way coupling solutions can be of second-order time accuracy and are more stable (see Vaassen et al. [11]). The one-way coupling method does not guarantee energy conservation at the interface, but two-way does. A benefit of one-way coupling simulation is significantly lower computational time. A second benefit is that deformation of the fluid mesh does not need to be calculated, which provides a mesh of constant quality.

\section{Mesh Deformation}

In two-way coupling calculations, the deformation of the boundary between fluid and solid deforms the fluid mesh. To calculate this deformation, the displacement diffusion algorithm is used. The connections between the nodes are modelled as springs, each with a specific stiffness. This stiffness can change from node to node; near boundaries, though, the mesh stiffness is set very high. In the normal direction from the fluid-structure boundary, the first ten nodes have displacements in the range of the boundary displacement. This provides high quality meshes even in the boundary layer. In Figure 2, a Nondeformed mesh is shown on the left, 


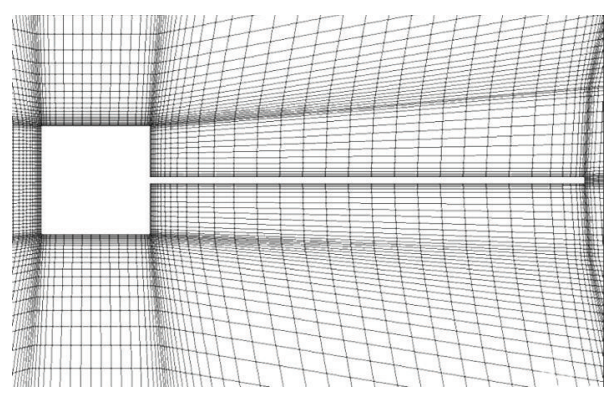

(a)

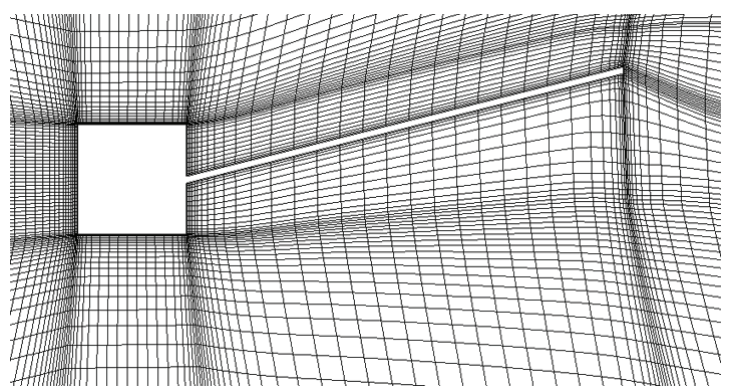

(b)

Figure 2: Nondeformed mesh compared to a deformed mesh.

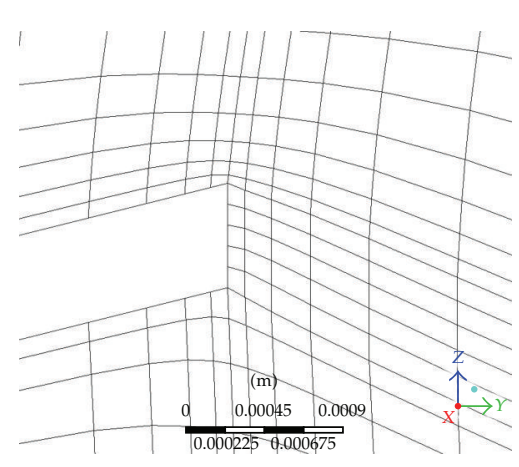

(a)

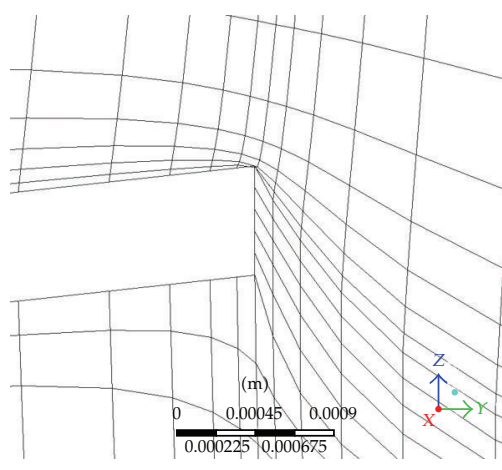

(b)

Figure 3: Different coefficients for mesh stiffness.

and a deformed mesh on the right. In both cases, the mesh has a satisfying quality. Figure 3 shows a deformed mesh of high quality on the left and a mesh of lower quality on the right.

\section{Simple Case for the Comparison between One- and Strong Two-Way Coupling}

The first test case is of very simple geometry and flow complexity. This benchmark case was originally published by Wall [14] and was also investigated by Walhorn [15] and Steindorf [16]. It consists of a square cylinder with a thin plate at the trailing side. The plate is influenced by the wake behind the cylinder. The flow regime can be changed by varying the inlet velocity. Two different materials are used for the plate, which leads to different natural frequencies. For a given inlet velocity, van Karman vortexes develop at the square cylinder. The shedding frequency is selected for both possibilities close to and further away from the natural frequencies of the plate. The investigated geometry, including its mesh, is shown in Figure 4. The different inlet velocities under investigation are shown in Table 1, and the natural frequencies of the plate are shown in Table 2. For evaluation of the results, the displacement of the trailing edge of the plate divided by the height of the square cylinder is computed over time. With a fast Fourier transformation (FFT), the frequencies of the oscillations are determined. Unfortunately, there are no experimental studies that have been done on this problem. Therefore, a comparison with the numerical results of Walhorn [15] is carried out. 


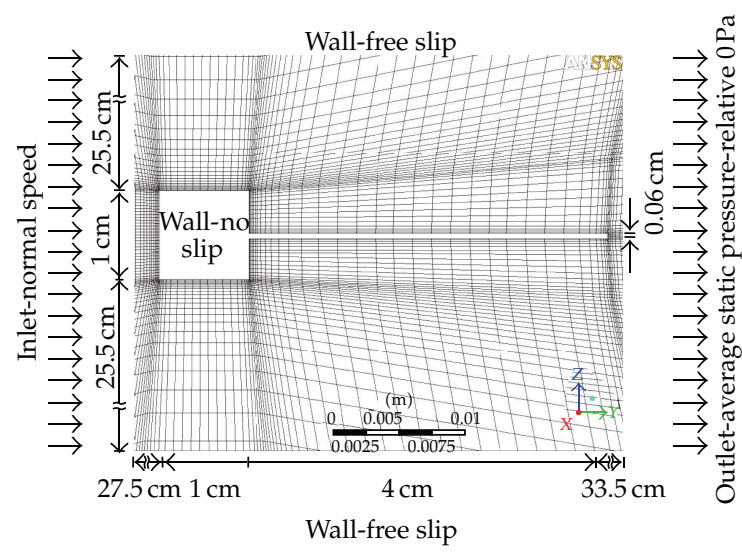

Figure 4: Simple case domain with boundary conditions.

Table 1: Investigated inlet conditions and material parameters.

\begin{tabular}{lccc}
\hline No. & Velocity $(\mathrm{m} / \mathrm{s})$ & Shedding frequency $(\mathrm{Hz})$ & Plate \\
\hline 1 & 0.27 & 3.02 & Plate 2 \\
2 & 0.34 & 3.68 & Plate 1 \\
3 & 0.34 & 3.68 & Plate 2 \\
4 & 0.64 & 7.03 & Plate 2 \\
\hline
\end{tabular}

Table 2: Natural frequencies of plates 1 and 2.

\begin{tabular}{lcc}
\hline$i$ & Natural frequency for plate $1(\mathrm{~Hz})$ & Natural frequency for plate $2(\mathrm{~Hz})$ \\
\hline 1 & 3.07 & 0.78 \\
2 & 15.13 & 3.03 \\
3 & 19.2 & 3.84 \\
4 & & 10.74 \\
5 & & 18.52 \\
\hline
\end{tabular}

\section{Case Number 1: Inlet Velocity $v=0.27 \mathrm{~m} / \mathbf{s}$ with Material Parameters of Plate 2}

In case Number 1, the vortex shedding frequency and the second natural frequency of the plate are identical; therefore, the plate should oscillate at this frequency. The displacement that occurs over time is shown in Figure 5, and the frequency spectrum of displacement is shown in Figure 6. However, the two-way coupling solution shows that the plate oscillates after a few seconds at the first natural frequency of $f=0.78 \mathrm{~Hz}$. The maximum displacement, calculated with the one-way coupling algorithm, shows a different behaviour in terms of displacement. The displacement of the plate is periodically constant after $5 \mathrm{~s}$, and the maximum displacement is one-fortieth the displacement that was calculated with the twoway coupling solution. The frequency spectrum does not show this big difference, and the mean frequency is the first natural frequency for both cases. In the one-way coupling solution, the second natural frequency is present, but with a low magnitude.

In Figure 7, the displacement of the plate is shown at $15 \mathrm{~s}$ for the one- (red) and twoway (black) coupling solution. 


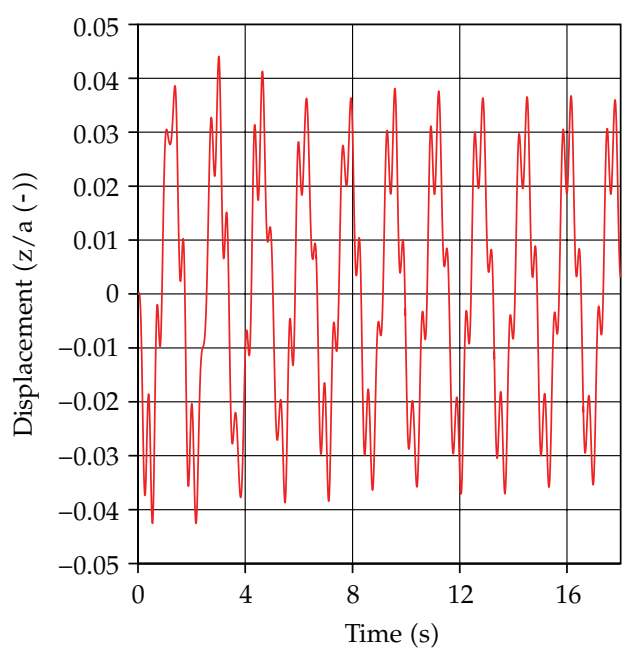

One-way coupling

(a)

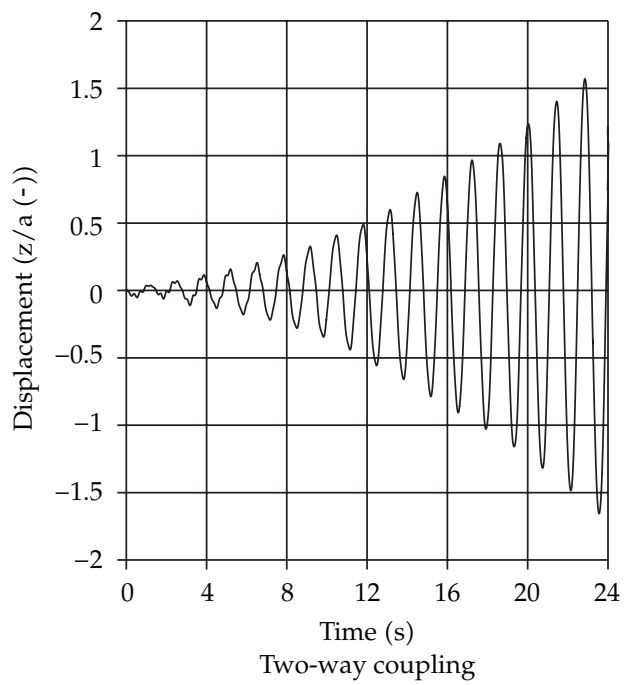

(b)

Figure 5: Displacement over time, $v=0.27 \mathrm{~m} / \mathrm{s}$, plate 2 .

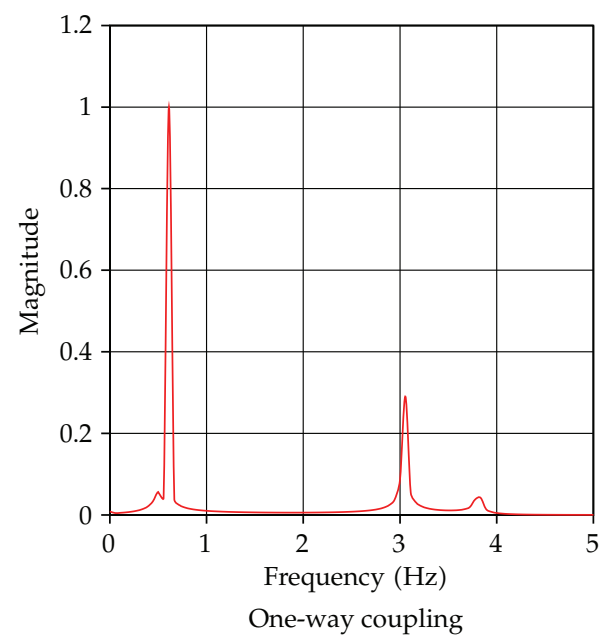

(a)

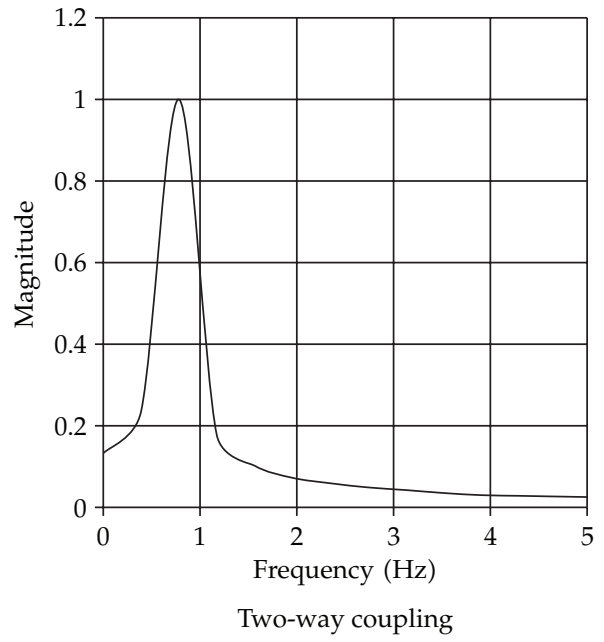

(b)

Figure 6: Frequency spectrum, $v=0.27 \mathrm{~m} / \mathrm{s}$, plate 2 .

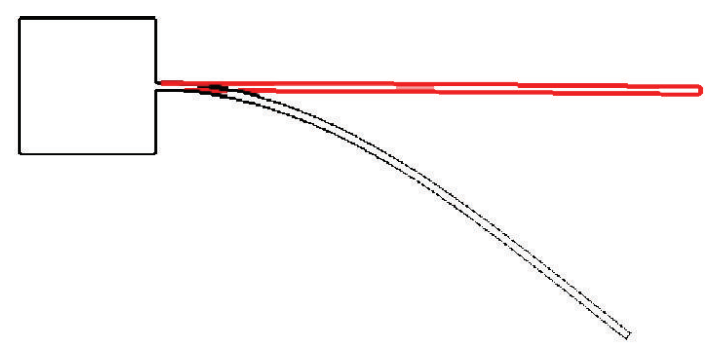

Figure 7: Comparison between deflections, $v=0.27 \mathrm{~m} / \mathrm{s}$, plate 2 . 


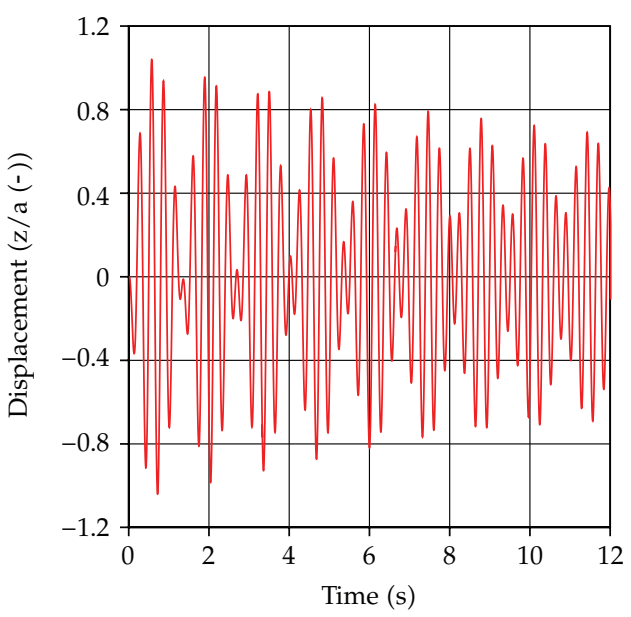

One-way coupling

(a)

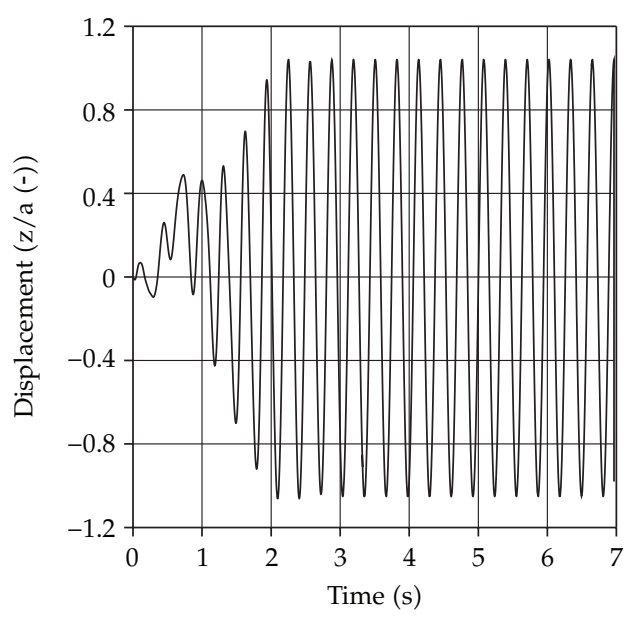

Two-way coupling

(b)

Figure 8: Displacement over time, $v=0.34 \mathrm{~m} / \mathrm{s}$, plate 1 .

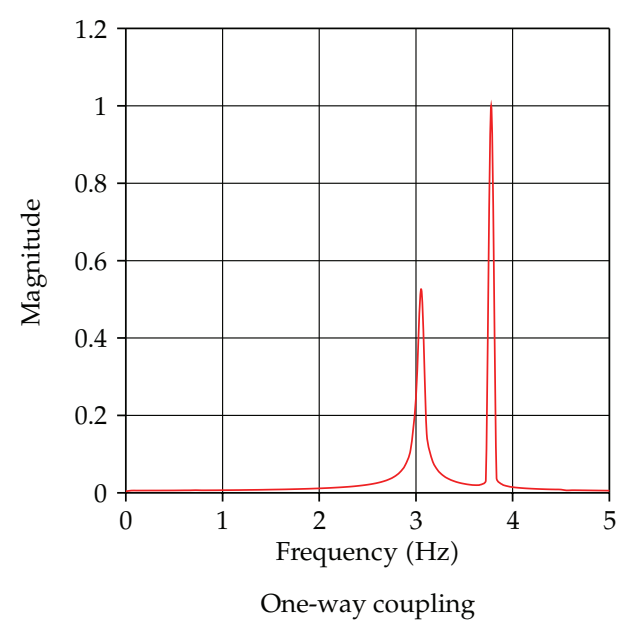

(a)

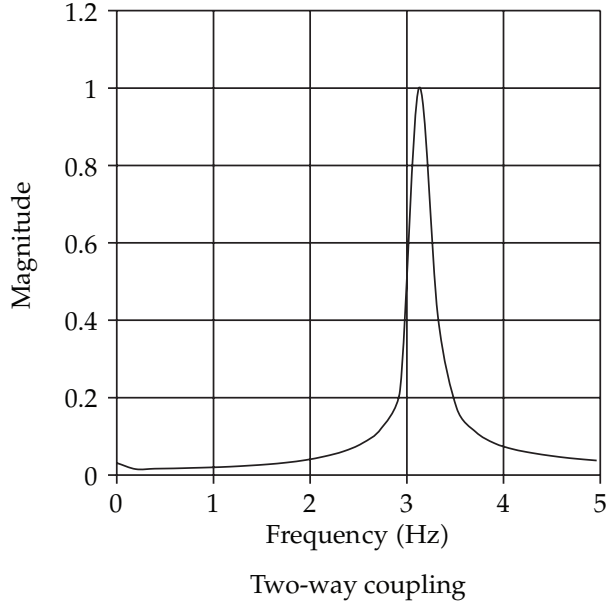

(b)

Figure 9: Frequency spectrum, $v=0.34 \mathrm{~m} / \mathrm{s}$, plate 1 .

\section{Case Number 2: Inlet Velocity $v=0.34 \mathrm{~m} / \mathbf{s}$ with Material Parameters of Plate 1}

In the second case, the vortex shedding frequency $f_{F}=3.68 \mathrm{~Hz}$ is close to the natural frequency of plate $1\left(f_{S}=3.07 \mathrm{~Hz}\right)$. After two seconds, the trailing edge displacement of the plate reaches a maximum of one and stays periodically constant in the two-way coupling solution. In the one-way coupling solution, the maximum displacement is also one in the first two seconds, but fades to 0.5 (see Figure 8). The frequency spectrum of the displacement shows only one frequency, $f=3 \mathrm{~Hz}$, which can be assigned to the first natural frequency for the two-way coupling case. The one-way coupling solution shows a second frequency, $f=3.7 \mathrm{~Hz}$, which can be assigned to the vortex shedding frequency (see Figure 9). 


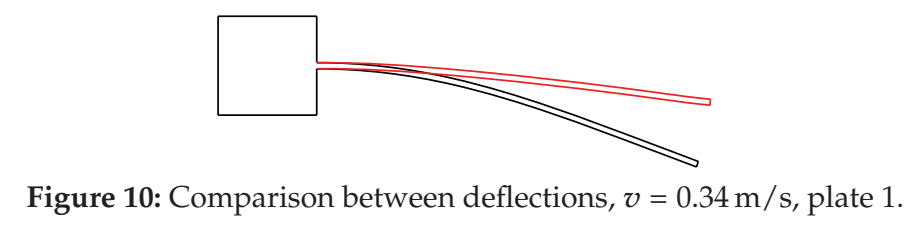

In Figure 10, the displacement of the plate is shown at $15 \mathrm{~s}$ for the one- (red) and twoway (black) coupling solution.

\section{Case Number 3: Inlet Velocity $v=0.34 \mathrm{~m} / \mathrm{s}$ with Material Parameters of Plate 2}

In case Number 3, the two-way coupling solution shows the expected behaviour near the structure's natural frequency. Starting with a small deflection, it grows in an exponential manner until a maximum displacement of 0.5 is reached, which happens in about $10 \mathrm{~s}$. In the one-way coupling solution, the deflection is also small at the beginning of the calculation, but increases to an averaged maximum of 0.25 . In both solution algorithms, the oscillating frequency is near the third natural frequency of the plate (see Figure 12). The two-way coupling solution shows good agreement with the numerical results from Walhorn [15], which was calculated with a monolithic approach (shown in Figure 11).

\section{Case Number 4: Inlet Velocity $v=0.64 \mathrm{~m} / \mathbf{s}$ with Material Parameters of Plate 2}

In this case, the vortex shedding frequency is far from the natural frequencies of plate 2 . The behaviour-based on the two-way coupling algorithm - is quite different compared to cases $1-3$. Here, the plate does not oscillate around zero, but instead vibrates around a mean value of 0.2 , as shown in Figure 13. The oscillating frequency of the plate is the vortex shedding frequency of $f_{F}=7.03 \mathrm{~Hz}$ (see Figure 14). The one-way coupling solution shows only a small, low-magnitude oscillation. The oscillating frequency is $0.78 \mathrm{~Hz}$, which is the first natural frequency.

\section{Summary of the Simple Case}

This simple case shows that the solutions for one- and two-way coupling yield very different results for the displacement. For cases in which the vortex shedding frequency is close to the natural frequency of the plate, the history of displacement can be compared between oneway and two-way coupling. In all cases in which the vortex shedding frequency is near the natural frequency of the plate, the mean frequency of oscillation is the same. In case Number 4 , where the vortex shedding frequency is at a distance from the natural frequency, the oneway coupling solution cannot be compared to the two-way coupling solution in terms of displacement magnitude or frequency of displacement.

\section{Investigation of a Single-Blade Sewage Pump}

Single-blade pumps are often used when a suspension of rough solids or cellulose in water has to be transported. Such a commercial single-blade pump is shown in Figure 15. 


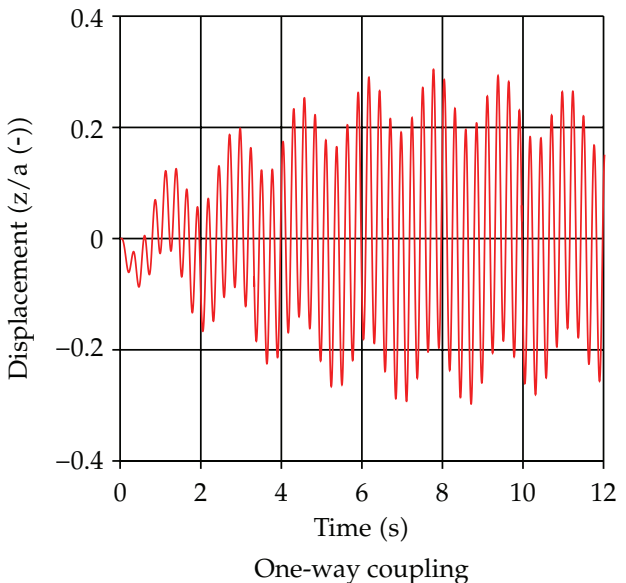

(a)

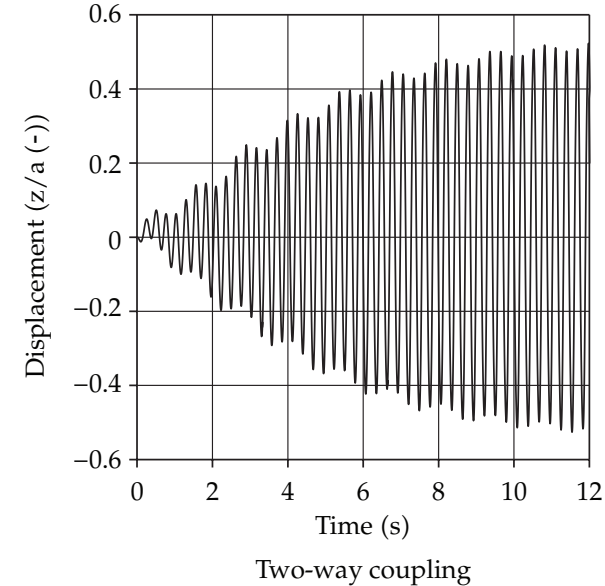

(b)

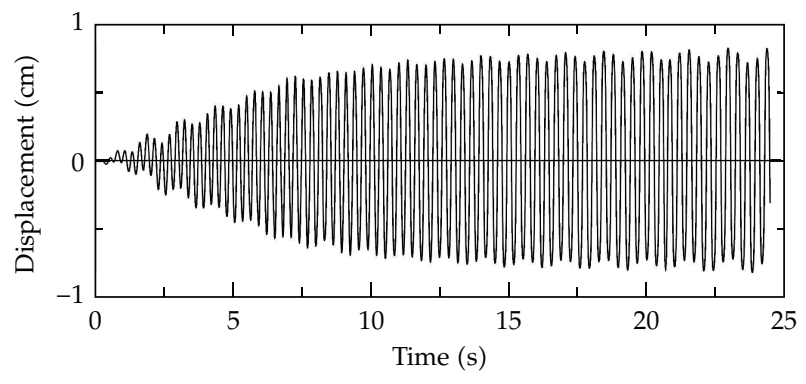

Result from Walhorn [15] with the studied vortex shedding frequency and plate 2

(c)

Figure 11: Displacement over time, $v=0.34 \mathrm{~m} / \mathrm{s}$, plate 2 .

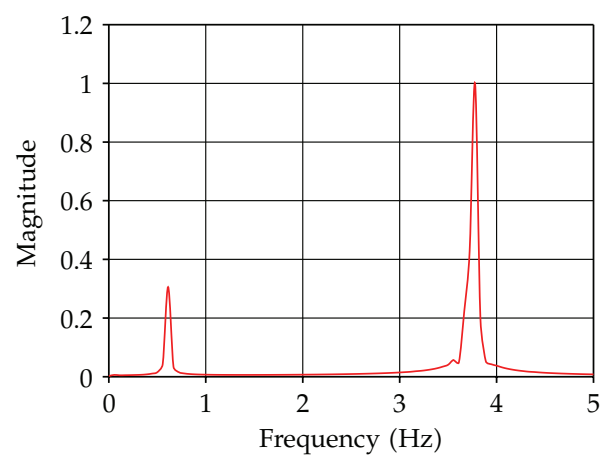

One-way coupling

(a)

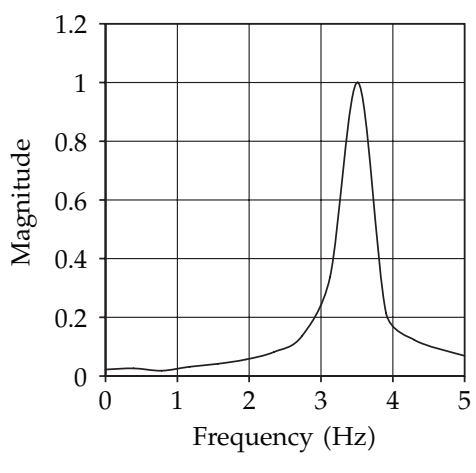

Two-way coupling

(b)

Figure 12: Frequency spectrum, $v=0.34 \mathrm{~m} / \mathrm{s}$, plate 2 .

Numerical and experimental investigations of single-blade pumps have been conducted at the University of Duisburg-Essen [18-22]. A detailed comparison between the simulation results of two-way coupling FSI and experimental results were published by Benra [23]. 


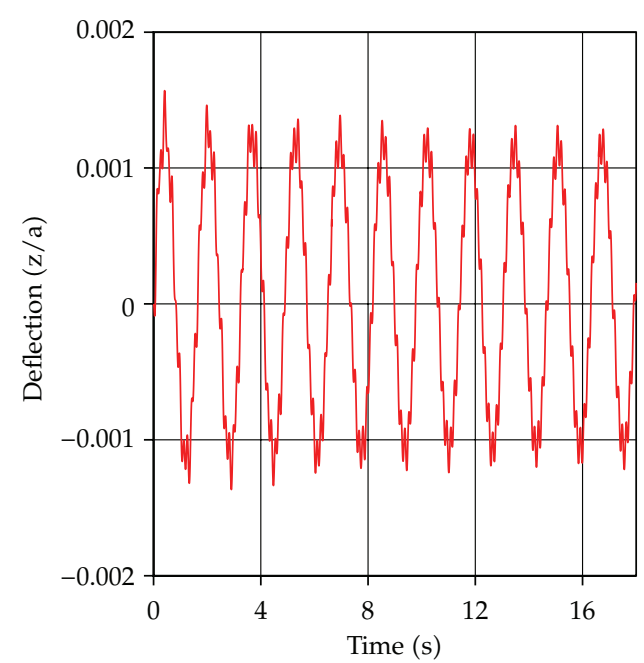

One-way coupling

(a)

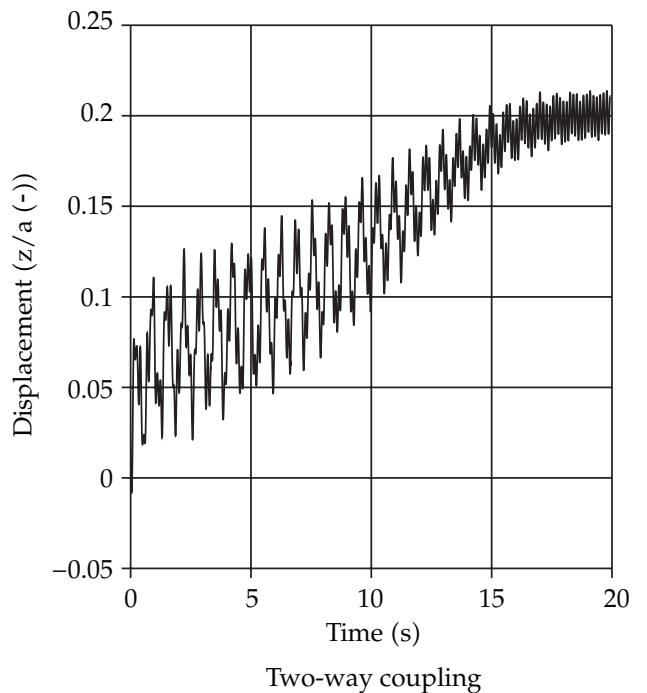

(b)

Figure 13: Displacement over time, $v=0.64 \mathrm{~m} / \mathrm{s}$, plate 2 .

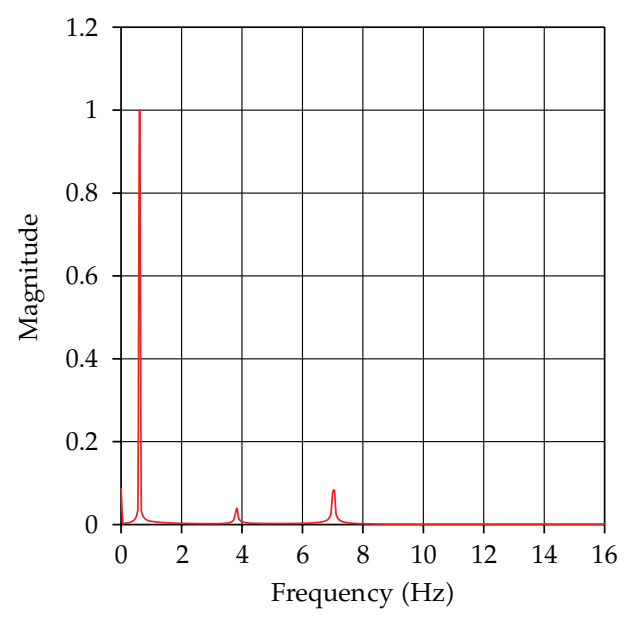

One-way coupling

(a)

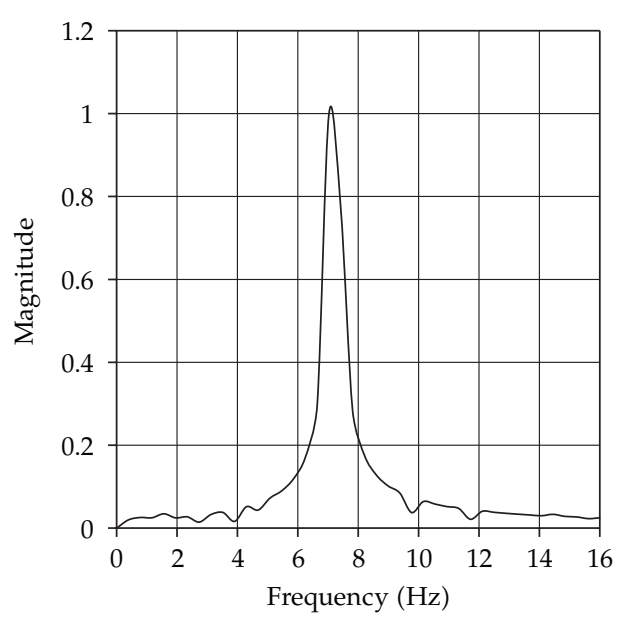

Two-way coupling

(b)

Figure 14: Frequency spectrum, $v=0.64 \mathrm{~m} / \mathrm{s}$, plate 2 .

A model of the pump for the FSI simulation is shown in Figure 16. Apart from the casing and impeller, part of the shaft was also taken into account for the flow simulation. For structural analysis, the complete shaft and impeller were considered. Keeping the correspondence of the coordinate system for both simulations, the values for load and mesh deformation were exchanged during the FSI calculation process.

In physics, an individual blade may cause an uneven mass distribution over the perimeter of an impeller. On the one hand, this leads to a stimulation of the vibrations with the frequency of the rotor revolution due to mechanical imbalances. On the other 


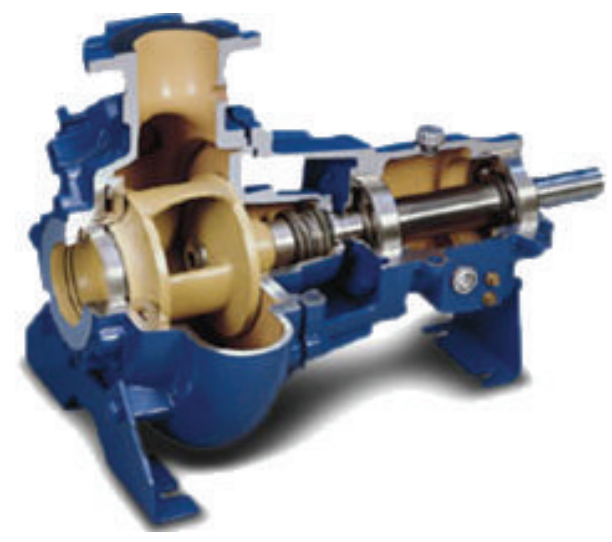

Figure 15: Commercial single-blade pump [17].
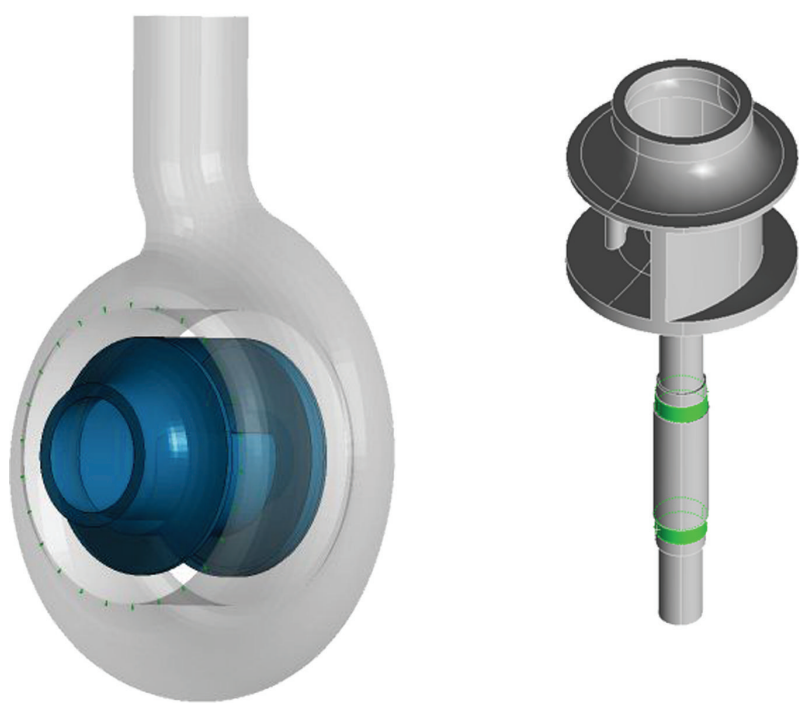

Figure 16: Flow calculation model and structural calculation model.

hand, the flow around the blade produces a different pressure field on the surfaces of the blade. The development of pressure around the suction surface leads to an uneven pressure distribution at the rotor outlet for a single-blade rotor. The interaction between the flow in the impeller and the casing during an impeller revolution results in a transient pressure field which is periodic with the rotor revolution. This leads to a variable radial force, which is indicated as a hydrodynamic imbalance. Mechanical and hydrodynamic imbalances produce radial deflections of the pump shaft that can be recognized as vibrations at bearing blocks. These vibrations will be transferred to the whole pumping set and equipment components.

A FSI simulation is required to show the complete effect of such vibrations. The impeller deflection changes the gap between the casing and impeller and strongly affects the flow field. Then, the redistributed pressure field disturbs the vibration orbit of the impeller, which generates a stronger impeller vibration than before. Such phenomena are described 


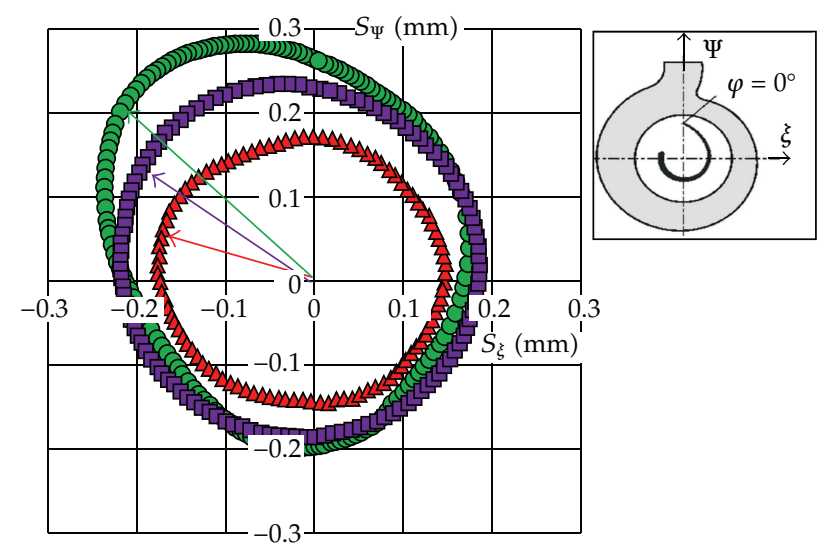

- Measurement at commercial sewage water pump

$\Delta$ One-way coupling: dynamic calculation (by forces)

$\square$ Weak TWC solution (by pressure field)

Figure 17: Impeller vibration orbit.

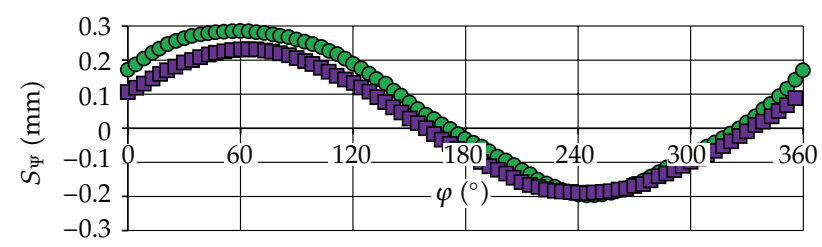

(a)

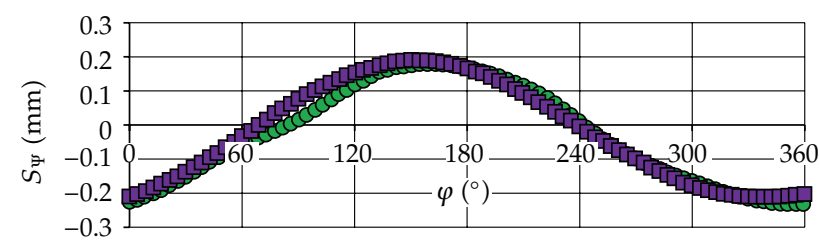

- Weak TWC solution

○ Measurement

(b)

Figure 18: Comparison of calculated and measured deflection.

in Figure 17, which gives a comparison between one-way and weak two-way coupled calculations. It is important to compare the results with the experimental data, as can be seen in Figure 17. The weak two-way coupled results show better agreement with the measurements, especially in the 1st to 3rd coordinate quadrants. This supports the previous analysis that the two-way coupled calculation offered results that were closer to physical observations.

A closer look at the two-way coupling simulation results and actual measurements shows good agreement. For a full $360^{\circ}$ rotation of the pump impeller, the deflection is shown in the $\Psi$ direction in the top picture; deflection is in the $\xi$ direction in the bottom picture (Figure 18). 


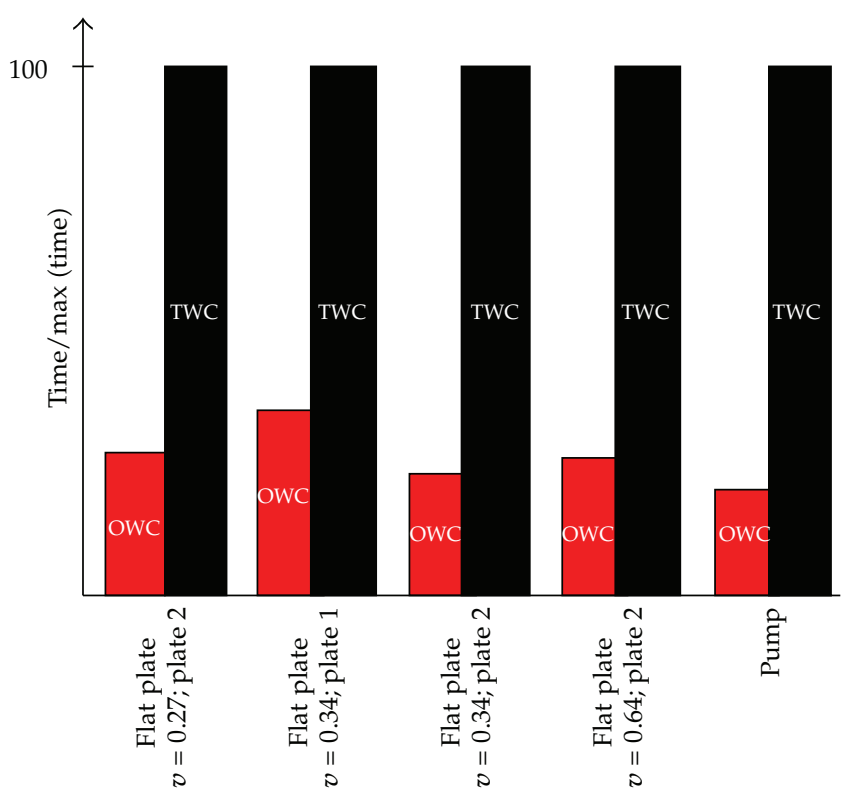

Figure 19: Solution time for one- and two-way coupling.

\section{Comparison of Solution Time}

In Figure 19, a comparison of the solution time between the two methods is shown. For every example, the same simulation times were compared. The additional time for a two-way coupling simulation is between three to four times the solution time for a one-way coupling simulation.

\section{Conclusion}

In this paper, problems concerning the interaction between fluids and structures were solved with one-way and two-way coupling methods. For a simple case, it was shown that the frequency of deflection was nearly the same for both methods if the vortex shedding frequency was close to the natural frequency. For every case, the deflection of the plate for the one-way coupling solution was much lower than it was for the two-way coupling solution. For the case where the vortex shedding frequency was far from the natural frequency, the results of the two algorithms were different in both frequency and deflection.

The results of the investigation for the single-blade pump showed significant differences between the one-way coupling solution and experimental results. The maximum deflection was under-predicted by this method. For all impeller positions, the impeller orbit curve showed nearly the same magnitude of displacement. The two-way coupling solution algorithm could predict the impeller orbit curve for a wide range, except for the high deflections in the 4th coordinate quadrant the method failed to predict the measured deflections. Overall, the two-way coupling method yielded results that were close to reality, whereas the one-way coupling algorithm gave plausible results only for specific values, in some cases. 


\title{
Nomenclature
}

\author{
Arabic and Greek Letters \\ $a$ : Height of square cylinder \\ $f$ : Frequency \\ $F$ : Force \\ $m$ : Mass \\ $p$ : Static pressure \\ $t$ : Time \\ $v$ : Velocity \\ $V$ : Volume \\ $Z$ : Deflection \\ $\Omega$ : Domain \\ $\rho$ : Density \\ $\Gamma$ : Boundary
}

Indices/Superscripts

$\begin{array}{ll}I: & \text { Interface } \\ F: & \text { Fluid } \\ S: & \text { Structure } \\ x, y, z: & \text { Cartesian coordinates. }\end{array}$

\section{Abbreviations}

FSI: Fluid-structure interaction

OWC: One-way coupling

TWC: Two-way coupling.

\section{References}

[1] O. C. Zienkiewicz and R. L. Taylor, The Finite Element Method, vol. 1, Butterworth-Heinemann, 5th edition, 2000.

[2] M. Schäfer, G. Sieber, R. Sieber, and I. Teschauer, "Coupled fluid-solid problems: examples and reliable numerical simulation," in Proceedings of the Trend in Computational Structural Mechanics, International Center for Numerical Methods in Engineering (CIMNE), Barcelona, Spain, May 2001.

[3] K. C. Park, C. A. Felippa, and J. A. DeRuntz, "Stabilization of staggered solution procedures for fluid-structure interaction analysis," in Computational Methods for Fluid-Structure Interaction Problems, T. Belytschko and T. L. Geers, Eds., vol. 26, pp. 95-124, American Society of Mechanical Engineers, New York, NY, USA, 1977.

[4] C. A. Felippa and K. C. Park, "Staggered transient analysis procedures for coupled mechanical systems: formulation," Computer Methods in Applied Mechanics and Engineering, vol. 24, no. 1, pp. 61$111,1980$.

[5] S. Piperno, C. Farhat, and B. Larrouturou, "Partitioned procedures for the transient solution of coupled aeroelastic problems, part I: model problem, theory and two-dimensional application," Computer Methods in Applied Mechanics and Engineering, vol. 124, no. 1-2, pp. 79-112, 1995.

[6] S. Piperno, "Explicit/implicit fluid/structure staggered procedures with a structural predictor and fluid subcycling for 2D inviscid aeroelastic simulations," International Journal for Numerical Methods in Fluids, vol. 25, no. 10, pp. 1207-1226, 1997.

[7] D. P. Mok, W. A. Wall, and E. Ramm, "Accelerated iterative substructuring schemes for instationary fluid structure interaction," in Computational Fluid and Solid Mechanics, K. J. Bathe, Ed., pp. 1325-1328, Elsevier, 2001. 
[8] F. Nobile, Numerical approximation of fluid structure interaction problems with application to haemodynamics, Ph.D. thesis, EPFL, Switzerland, 2001.

[9] D. Peric and W. G. Dettmer, "A computational strategy for interaction of fluid flow with spatial structures," in Proceedings of the 5th International Conference on Computational of Shell E Spatial Structures (IASS IACM '05), 2005.

[10] A. Timperi, "Fluid-structure interaction calculations using a linear perturbation method," in Proceedings of the 20th International Conference on Structural Mechanics in Reactor Technology (SMiRT '09), 2009.

[11] J.-M. Vaassen, P. DeVincenzo, C. Hirsch, and B. Leonard, "Strong coupling algorithm to solve fluidstructure-interaction problems with a staggered approach," in Proceedings of the 11th International WS on Simulation E EGSE Facilities for Space Programmes (SESP '10), 2010.

[12] A. Pozarlik and J. Kok, "Numerical investigation of one- and two-way fluid-structure interaction in combustion systems," in Proceedings of the International Conference on Computational Methods for Coupled Problems in Science and Engineering, 2007.

[13] M. Münsch and M. Breuer, "Numerical simulation of fluid-structure interaction using eddy-resolving schemes," in Fluid Structure Interaction II, H.-J. Bungartz, M. Mehl, and M. Schäfer, Eds., Lecture Notes in Computational Science and Engineering, pp. 221-254, Springer, Berlin, Germany, 2010.

[14] W. Wall, Fluid-struktur interaktion mit stabilisierten finiten elemente, Dissertation, Stuttgart, Germany, 1999.

[15] E. Walhorn, Ein simulatenes berechnungsverfahren für fluid-struktur-wechselwirkung mit finiten raum-zeitelementen, Dissertation, Braunschweig, Germany, 2002.

[16] J. Steindorf, Partitionierte verfahren für probleme der fluid-struktur wechselwirkung, Dissertation, Braunschweig, Germany, 2002.

[17] RITZ Pumpenfabrik GmbH \& Co. KG, “Kanalradpumpe 38," 2011, http:/ /www.ritz-pumpen.de/.

[18] F.-K. Benra and H. J. Dohmen, "Investigation on the time-variant flow in a single-blade centrifugal pump," in Proceedings of the 5th WSEAS International Conference on Fluid Mechanics (FLUIDS '08), Acapulco, Mexico, January 2008.

[19] F.-K. Benra and H. J. Dohmen, “Numerical and experimental evaluation of the time-variant flow field in a single-blade centrifugal pump," in Proceedings of the 5th International Conference on Heat Transfer, Fluid Mechanics and Thermodynamics (HEFAT '07), pp. 1-4, Sun City, South Africa, July 2007.

[20] F.-K. Benra, H. J. Dohmen, and M. Sommer, "Flow field visualization of a single-blade centrifugal pump using PIV-method-comparison to numerical results," Journal of Visualization, vol. 9, no. 4, p. $358,2006$.

[21] F.-K. Benra and N. Savilius, "Experimental determination of the hydrodynamic imbalance of a singleblade centrifugal pump," WSEAS Transactions on Fluid Mechanics, vol. 1, no. 6, pp. 707-713, 2006.

[22] F.-K. Benra and N. Savilius, "Experimental investigation of transient hydrodynamic forces of a singleblade centrifugal pump," in Proceedings of the 4th WSEAS International Conference on Fluid Mechanics and Aerodynamics, Crete Island, Greece, August 2006.

[23] F.-K. Benra, "Numerical and experimental investigation on the flow induced oscillations of a singleblade pump impeller," Journal of Fluids Engineering, vol. 128, no. 4, pp. 783-793, 2006. 


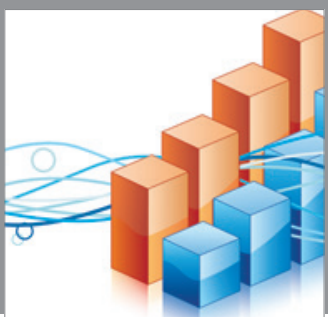

Advances in

Operations Research

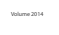

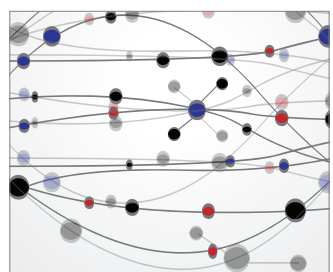

\section{The Scientific} World Journal
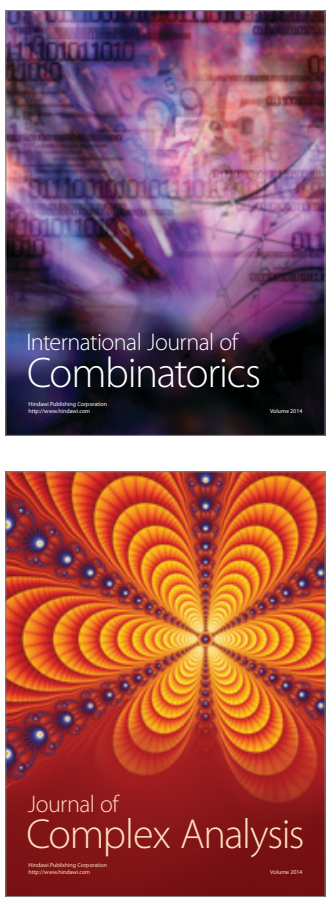

International Journal of

Mathematics and

Mathematical

Sciences
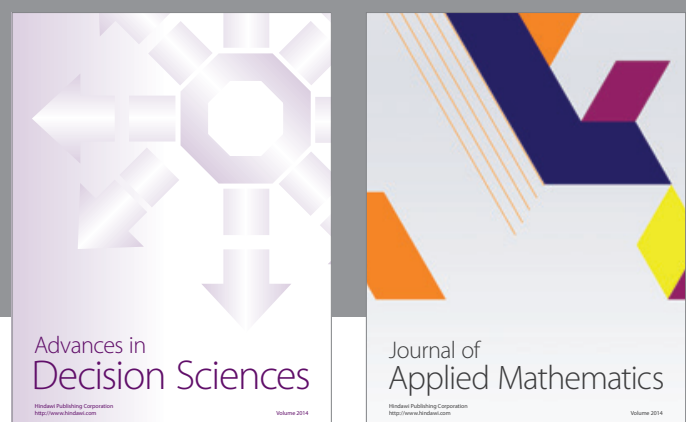

Journal of

Applied Mathematics
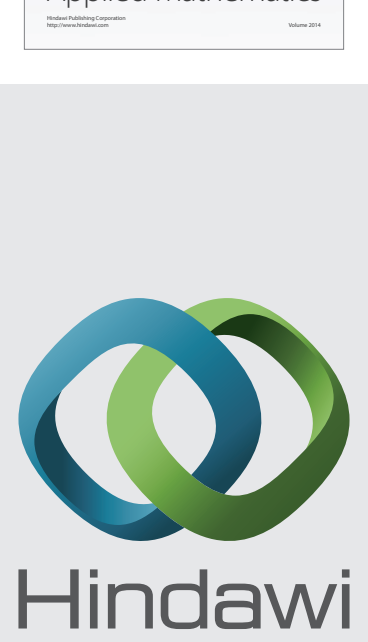

Submit your manuscripts at http://www.hindawi.com
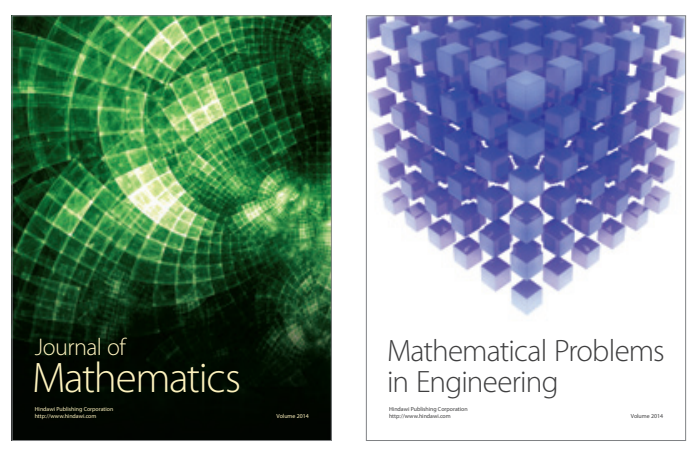

Mathematical Problems in Engineering
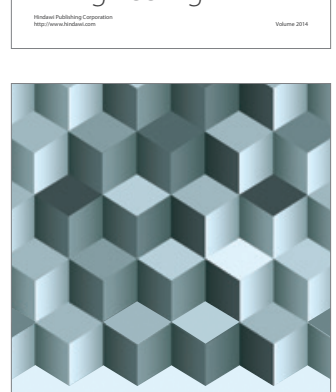

Journal of

Function Spaces
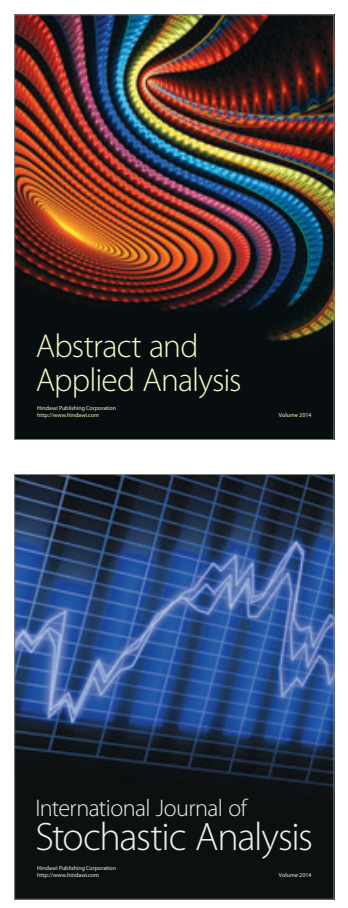

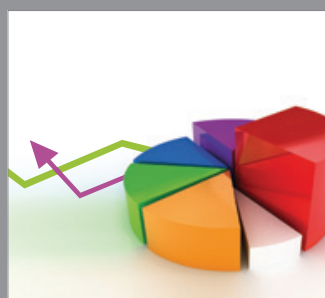

ournal of

Probability and Statistics

Promensencen
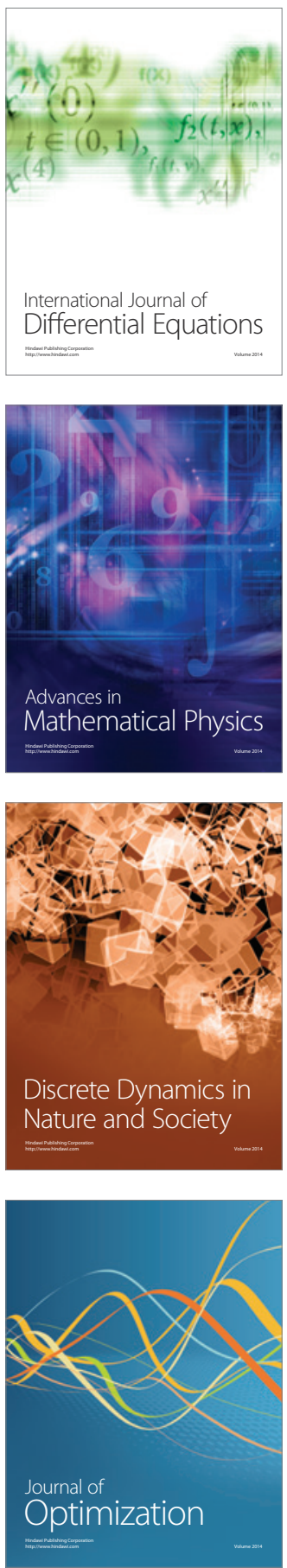